This is a post-print of the following article: Varvenne, C., Luque, A., Nöhring, W. G. \& Curtin, W. A. Average-atom interatomic potential for random alloys. Physical Review B 93, 104201 (2016). The final publication is available at http://dx.doi.org/10.1103/PhysRevB.93.104201 (C)American Physical Society

\title{
Average-atom interatomic potential for random alloys
}

\author{
Céline Varvenne ${ }^{*}$ Aitor Luque, Wolfram G. Nöhring, and William A. Curtin \\ EPFL, STI-IGM, Laboratory for Multiscale Mechanics Modeling, CH-1015 Lausanne, Switzerland
}

(Dated: February 19, 2016)

\begin{abstract}
An average-atom ( $A$-atom) embedded-atom-method potential for random multicomponent alloys at any composition is derived analytically and validated by comparing $A$-atom and true random alloys bulk and defect properties, in model Fe-Ni-Cr systems. The $A$-atom can be mixed with the individual alloying-element potentials, thus enabling computation of defect/defect interactions. Its use provides quantitative insight into the physical role of the fluctuations, and has many applications, such as in atomistic/continuum modeling of random alloys and the development of new potentials with controlled properties.
\end{abstract}

\section{INTRODUCTION}

Atomistic simulations using semi-empirical potentials play a key role in uncovering mechanisms of deformation, diffusion, and phase transformation/growth in many materials 1 . In understanding the mechanical properties of metals, these atomistic simulations are particularly valuable because the behavior is controlled by interactions among defects, especially the interaction of dislocations with other defects like solutes, grain boundaries, precipitates, other dislocations or cracks, that involves scales often not reachable by ab initio methods. Most metals used in applications are multicomponent alloys, often non-dilute, which present additional complexity. Molecular Dynamics (MD) or Monte Carlo simulations require accurate interatomic potentials, and developing robust potentials remains a major challenge ${ }^{6}-19$, particularly for many-component alloys. Furthermore, in nondilute alloys, fluctuations in local atomic chemical environments make the analysis of important defect/defect interactions difficult and ill-defined. These local chemical fluctuations can, moreover, control some crucial properties of the alloy, such as the plastic flow stress. Separating the effects of overall composition from local fluctuations, and assessing which properties are controlled by fluctuations and which are not, requires additional tools and concepts.

To address the atomistic modeling of random alloys at arbitrary composition, we study in this article the concept of an "average-atom" ( $A$-atom) interatomic potential. We consider random alloys, i.e. systems where there is random occupation of the constituent atoms among arbitrary (non lattice) sites. Starting from a known set of embedded atom method (EAM) interatomic potentials for the multicomponent alloy system, we perform an analytic average over all possible random occupations of the atomic sites at the overall alloy composition and thereby define a single-atom "average-atom" potential meant to capture the average properties of the real disordered alloy. This concept was originally introduced by Smith et $a l .20$ and then applied to a few specific problems, such as reverse-engineering interatomic potentials using bulk properties from experiments $20 \mid 21$, modeling equilibrium segregation around defects 2223 , and studies of average dislocation core structures 24|25. Careful comparison of the method with true random alloys EAM results has not been done, so no assessment of its range of validity exists. Due to increasing computational power, the approach was largely been superseded by direct studies on true random configurations of alloys. Thus, features of the $A$-atom approach that remain useful and important for current research have never previously been identified.

In this paper, we re-derive and carefully validate the $A$-atom EAM potential concept for studies of bulk and defects properties, and highlight a number of the important but unrecognized features of the method. In particular, we demonstrate that the $A$-atom potential can be combined with any of the original atom potential, enabling study of the complex "average" defect/defect interactions that are nearly impossible to extract from direct simulations on explicit random alloys at high concentration. As computational metallurgy enters a new phase of mechanistic and predictive modeling in realistic alloys, applications of the $A$-atom potential to study a wide range of defects and mechanical properties in multicomponent random alloys, such as High Entropy Alloys and austenitic stainless steels for instance, will increase rapidly.

\section{PRE-AVERAGING PROCEDURE}

\section{A. Effective medium for the random alloy}

The basis theoretical framework strongly resembles effective-medium, Virtual Crystal Approximation (VCA) and Coherent Potential Approximation (CPA) approaches to alloys within electronic structure theory 26 - 33 . We consider an $N$-component alloy, with average concentration $c_{X}$ of each alloying element $X$, with $\sum_{X=1}^{N} c_{X}=$ 1. A configuration of the alloy consists of a set of atomic sites $\{i\}$ occupied by individual atoms, as denoted by site occupation variables $s_{i}^{X}$, with $s_{i}^{X}=1$ if a type- $X$ atom sits in $i$ site and 0 otherwise. Within the Embedded Atom 
Method $(\mathrm{EAM})^{\sqrt{6}}$, the energy of a given configuration is

$$
\begin{aligned}
& E\left(\left\{s_{i}^{X}\right\}\right)=\sum_{i, X} s_{i}^{X} F^{X}\left(\rho_{i}\right)+\frac{1}{2} \sum_{\substack{i, j \neq i \\
X, Y}} V_{i j}^{X Y} s_{i}^{X} s_{j}^{Y}, \\
& \text { with } \rho_{i}=\sum_{j \neq i, X} s_{j}^{X} \rho_{i j}^{X},
\end{aligned}
$$

where sums are over all atomic sites and $X, Y$ indicate the different individual atom types. As usual, $V_{i j}^{X Y}$ is the pairwise interaction between atoms $X$ and $Y$ and $F^{X}\left(\rho_{i}\right)$ is the embedding energy for atom $X$ at site $i$, with the local electron density $\rho_{i}$ at site $i$ generated by atoms surrounding site $i$. For a given set of atomic positions $\{i\}$, the average energy of the configuration is obtained by averaging over all possible site occupations consistent with the overall alloy composition. Since $F^{X}\left(\rho_{i}\right)$ is independent of the chemical occupation at site $i$ and since the occupations in sites $i$ and $j$ are uncorrelated in the random solid solution, the average energy can be written as

$$
\left\langle E_{0}\right\rangle=\sum_{i, X} c_{X}\left\langle F^{X}\left(\rho_{i}\right)\right\rangle+\frac{1}{2} \sum_{\substack{i, j \neq i \\ X, Y}} V_{i j}^{X Y} c_{X} c_{Y},
$$

where $\left\langle s_{i}^{X}\right\rangle=c_{X}$. We then perform a Taylor expansion $\left\langle F^{X}\left(\rho_{i}\right)\right\rangle=\left\langle F^{X}\left(\bar{\rho}_{i}\right)\right\rangle+O\left(\rho_{i}-\bar{\rho}_{i}\right)^{2}$ around the average electron density $\bar{\rho}_{i}$, in which the first order term vanishes since $\left\langle\left(\rho_{i}-\bar{\rho}_{i}\right)\right\rangle=0$. Neglecting second and higher-order terms ${ }^{34}$, which is the only approximation, the average energy is

$$
\begin{aligned}
\left\langle E_{0}\right\rangle & =\sum_{i} F^{A}\left(\bar{\rho}_{i}\right)+\frac{1}{2} \sum_{i, j \neq i} V_{i j}^{A A}, \\
\text { with } \quad F^{A}\left(\bar{\rho}_{i}\right) & =\sum_{X} c_{X} F^{X}\left(\bar{\rho}_{i}\right) ; \\
V_{i j}^{A A} & =\sum_{X, Y} c_{X} c_{Y} V_{i j}^{X Y} ; \bar{\rho}_{i}=\sum_{j \neq i} \sum_{X} c_{X} \rho_{i j}^{X},
\end{aligned}
$$

where $A$ denotes an "average-atom" having embedding function $F^{A}$ and self-pair-interaction $V_{i j}^{A A}$. The $A$-atom interatomic potential of Eq. 4 has exactly the EAM form for the potential energy of an elemental atom ${ }^{35}$, and so the $A$-atom is now a new atomic species that embeds the average properties of the random alloy. Note that all local fluctuations are averaged out analytically, and that the average is performed on any arbitrary set of atomic sites. The above derivation equally applies to Finnis-Sinclair ${ }^{7}$ and Second Moment Approximation ${ }^{8}$, and could be extended to modified EAM potentials $\frac{17 / 18}{}$.

\section{B. Constituent substitutional impurity}

The $A$-atom EAM potential can further be used in combination with the individual alloying elements $\{X\}$ in simulations, as if the A-atom is a new species. We can then compute, using standard definitions, the average properties of a substitutional "solute" atom of type $X_{0}$ in the $A$-atom host matrix, where $X_{0}$ is any of the constituent alloying elements. These properties require computing the average energy change when a solute $X_{0}$ is added to the random alloy. In a true random alloy, the energy change for atom $X_{0}$ is computed by replacing one other atom type $Y$ by an $X_{0}$ atom, computing the total energy change, repeating this procedure over many other atoms $Y$ in the structure, and then averaging these energies (see appendix A 3). This process can be performed analytically as follows. For a random configuration described by occupation variables $\left\{s_{i}^{X}\right\}$ and having energy $E\left(\left\{s_{i}^{X}\right\}\right)$, the atom at site $k$ is replaced by a type- $X_{0}$ atom, so that its occupation variables become $\tilde{s}_{k}^{X}$. The associated energy change is

$$
\Delta E=\sum_{X}\left(\tilde{s}_{k}^{X}-s_{k}^{X}\right) F^{X}\left(\rho_{k}\right)+\sum_{\substack{i \neq k \\ X, Y}} V_{k i}^{X Y} s_{i}^{Y}\left(\tilde{s}_{k}^{X}-s_{k}^{X}\right)(6)
$$

Averaging Eq. 6 over all possible atomic occupation configurations, using the Taylor expansion for the $F^{X}$ and the identities $\left\langle s_{i}^{X}\right\rangle=c_{X}$ for all $i$ and $\left\langle\tilde{s}_{k}^{X}\right\rangle=1$ if $X=X_{0}$ and 0 otherwise, the average energy change is

$$
\begin{gathered}
\langle\Delta E\rangle=\left[F^{X_{0}}\left(\bar{\rho}_{k}\right)+\sum_{i \neq k} V_{k i}^{X_{0} A}\right]-\left[F^{A}\left(\bar{\rho}_{k}\right)+\sum_{i \neq k} V_{k i}^{A A}\right] \\
\text { with } \quad V_{k i}^{X_{0} A}=\sum_{Y} c_{Y} V_{k i}^{X_{0} Y} .
\end{gathered}
$$

Here, $V_{k i}^{X_{0} A}$ is the pairwise interaction between $X_{0}$ and $A$ atoms. Eq. 7 is identical to the result obtained by introducing an atom $X_{0}$ into site $k$ of the $A$-atom EAM material, and is again independent of the atomic configuration $\{i\}$. Discussion of the many advantages of the $A$-atom potential is relegated to Sec. IV]

\section{AVERAGE-ATOM VERSUS TRUE RANDOM ALLOYS}

To be useful, the $A$-atom potential must be quantitatively accurate, which requires testing over a wide range of true random alloy properties. The averaging process eliminates the local variations in chemical occupations and the related local lattice relaxations that exist in the true random alloy, and so energies and forces in the $A$-atom material will only be approximate. Optimized structures and energies might thus differ between the $A$ atom and true random alloys. Here, we consider properties ranging from the bulk properties of the crystalline material to various defect structures and energies. As a model material, we use the fcc Fe-Ni-Cr ternary system, described with an EAM potentia $\sqrt{36}$. This allows a systematic comparison between true random alloys and their $A$-atom counterparts, and thus the accuracy of this Fe-Ni-Cr potential for any real alloy is irrelevant. We will 
often focus on $\mathrm{Fe}_{(1-x) / 2} \mathrm{Ni}_{(1-x) / 2} \mathrm{Cr}_{x}$ alloys for different $x$ values because our studies reveal that the $\mathrm{Cr}$ content controls many important property variations. All molecular statics computations reported here use standard methods executed within the open-source code LAMmP\$37.

\section{A. Random alloy properties}

We first briefly mention comparisons for bulk properties. The lattice parameters $a_{0}$ and cohesive energies $E_{\text {coh }}$ vs. $\quad$ Cr content $x$ for different fcc $\mathrm{Fe}_{(1-x) / 2} \mathrm{Ni}_{(1-x) / 2} \mathrm{Cr}_{x}$ materials, as computed for the $A$ atom and true random alloys (using $30 \times 30 \times 30 \mathrm{fcc}$ unit cells), are shown in Figs. 19,b. Agreement is excellent $(<2 \%)$. The shear elastic constants $C_{44}$ and $C^{\prime}=\left(C_{11}-C_{12}\right) / 2$ of the Fe-Ni-Cr ternary system over the entire composition range are provided in Figs. $2 \mathrm{a}, \mathrm{b}$, along with the absolute differences between $A$-atom and true random alloys (from Ref. 36, calculations performed on $5 \times 5 \times 5$ fcc unit cells). The agreement is again very good, with typical accuracy of $\sim 3 \%$ but occasionally larger differences (in any case, differences are $<5 \%$ for $C_{44}$, and $<15 \%$ for $C^{\prime}$ ) which may be, in part, due to fluctuations in the finite-size random alloys themselves.

We now consider planar defects, which involve additional atomic relaxations compared to the bulk. Planar defect energies, such as surface and stacking fault energies are relevant for fracture and dislocation structure/plasticity for instance ${ }^{38 / 39}$. Fig. 11. shows the surface energies $\sigma_{001}$ and $\sigma_{111}$ of the (001) and (111) planes, respectively, for the $A$-atom and true random materials for the $\mathrm{Fe}_{(1-x) / 2} \mathrm{Ni}_{(1-x) / 2} \mathrm{Cr}_{x}$ alloys (see Appendix A 1 for simulation details). Excellent agreement is obtained $(<1 \%)$. Fig. 20 shows the intrinsic stacking fault energies $\gamma_{\text {ISF }}$ across the full range of $\mathrm{Fe}-\mathrm{Ni}$-Cr compositions as computed using the $A$-atom potential along with the absolute differences relative to the true random alloys. The absolute differences are typically small, less than $10 \mathrm{~mJ} / \mathrm{m}^{2}$. Relative differences can be larger, $\approx 15 \%$, since $\gamma_{\mathrm{ISF}}$ itself is at least an order of magnitude smaller than the surface energies. Furthermore, overall trends with alloy composition are captured very well.

We next consider the dislocation, a line defect. We study the glissile $\{111\}(110)$ edge dislocation responsible for plasticity in fcc materials, which dissociates into two Shockley partials separated by an intrinsic stacking fault. The equilibrium separation distance $d_{\text {eq }}$ between the partials should be accurately described ${ }^{38}$ by the $A$ atom, since it accurately predicts the elastic constants and the stacking fault energy. Using standard techniques, we insert an initially-dissociated dislocation into each $\mathrm{Fe}_{(1-x) / 2} \mathrm{Ni}_{(1-x) / 2} \mathrm{Cr}_{x} A$-atom material, relax the structure, and measure $d_{\text {eq }}$. This procedure is repeated for the true alloys, starting from the partial separation measured in the $A$-atom materials (see Appendix A 2). After relaxation, the partial dislocations in the random alloys glide small distances and adopt wavy configurations, both due

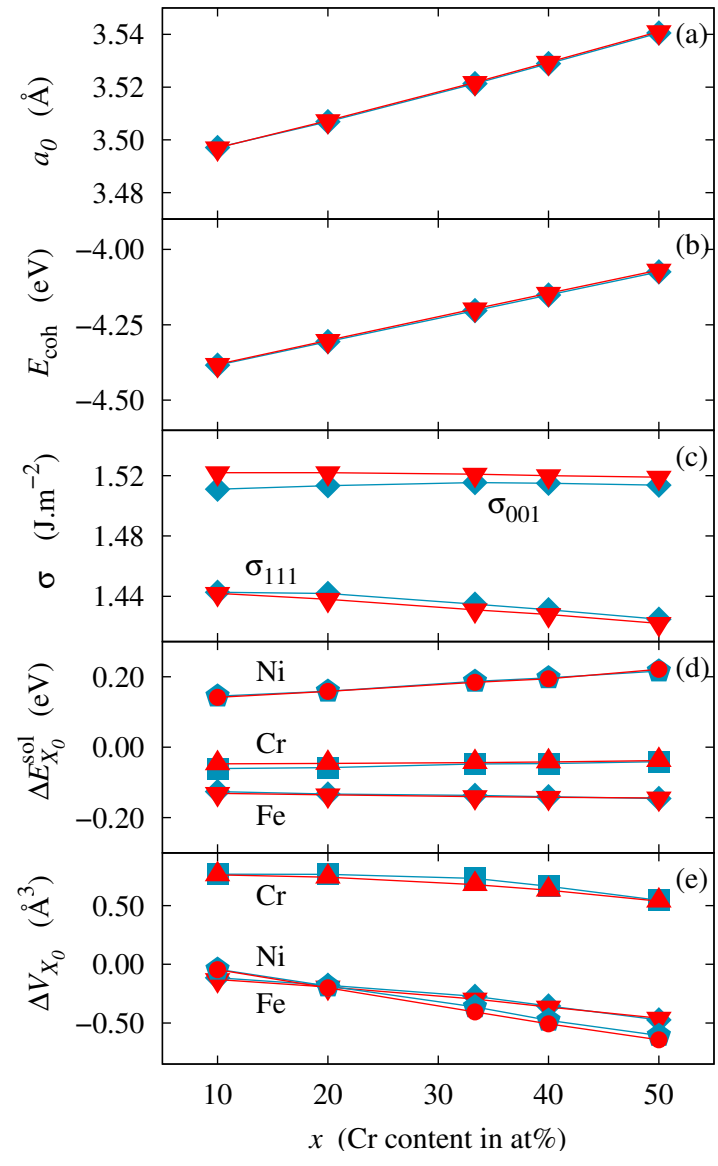

FIG. 1. Properties of $\mathrm{Fe}_{(1-x) / 2} \mathrm{Ni}_{(1-x) / 2} \mathrm{Cr}_{x}$ alloys vs. $\mathrm{Cr}$ content $x$ : (a) lattice parameter $a_{0}$ (b) cohesive energies $E_{\text {coh }}$, (c) surface energies $\sigma_{001}$ and $\sigma_{111},(d)$ solute energies $E_{X_{0}}^{\text {sol }}$ and (e) solute misfit volumes $\Delta V_{X_{0}}$, measured in $A$-atom (red) and true random (blue) alloys.

to local compositional/structural fluctuations that interact with the dislocation stress field. As shown in Fig. 3. the average partial dissociation distances in the true random alloys, averaged over 20 different realizations at each composition, match well with those predicted by the $A$ atom materials for $x=0.4$ and 0.5 but deviate somewhat for lower $x$. The local fluctuations along the line defect in the true random alloy exert forces on the dislocation line that cause deviations relative to the $A$-atom material, even though the two materials have essentially equal average material properties. This demonstrate the role of the fluctuations in controlling the very detailed dislocation configuration. More important is the glide stress. Although not shown, the glide stresses in the $A$-atom materials are very low $(<10 \mathrm{MPa})$, as expected for elemental fcc metals (e.g. Al, $\mathrm{Ni}, \mathrm{Cu})$. In contrast, the glide stresses in the true random alloys are very large $(\sim 200$ $500 \mathrm{MPa}$, depending on dislocation line length and random sample), due to the interactions of the dislocation with the local chemical and structural fluctuations in the true random alloy. Thus, the plastic flow stress is con- 
This is a post-print of the following article: Varvenne, C., Luque, A., Nöhring, W. G. \& Curtin, W. A. Average-atom interatomic potential for random alloys. Physical Review B 93, 104201 (2016). The final publication is available at http://dx.doi.org/10.1103/PhysRevB.93.104201 (C)American Physical Society

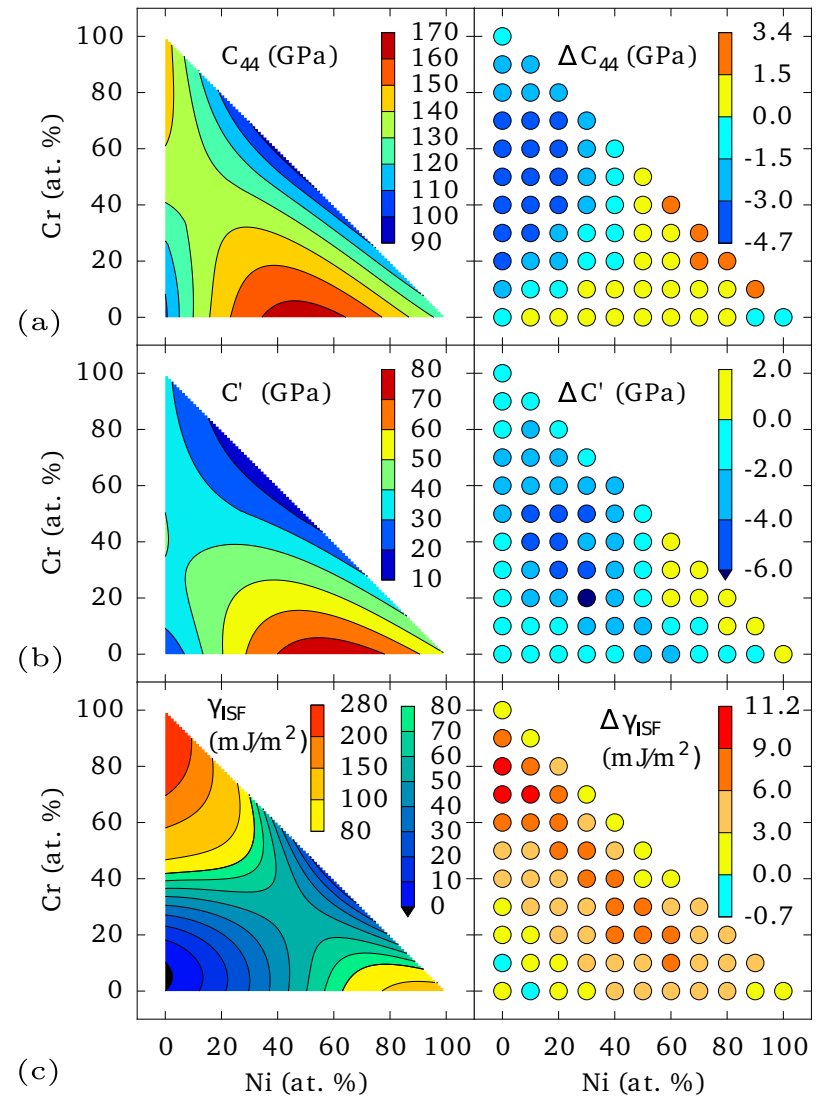

FIG. 2. Shear elastic constants (a) $C_{44}$ and (b) $C^{\prime}=$ $\left(C_{11}-C_{12}\right) / 2$, and $(\mathrm{c})$ intrinsic stacking fault energy $\gamma_{\mathrm{ISF}}$, as computed with the $A$-atom potential (left), along with the absolute differences relative to the true random alloys $\frac{36}{\text { (right), }}$ versus composition of the Fe-Ni-Cr ternary alloys. The dark blue point in $\Delta C^{\prime}$ has the value $-10.4 \mathrm{GPa}$.

trolled by fluctuations and cannot be modeled using the $A$-atom material; the mechanistic origins of the strengthening in the true random alloys are discussed elsewhere 4 .

\section{B. Constituent "solute" properties}

We now investigate the accuracy of the mixing of $A$-atoms with true atoms to compute real atom "solute" properties in the alloy. Specifically, we compute the solute energies $\Delta E_{X_{0}}^{\mathrm{sol}}$ and misfit volumes $\Delta V_{X_{0}}$ for $X_{0}=\mathrm{Fe}, \mathrm{Cr}, \mathrm{Ni}$ in the $\mathrm{Fe}_{(1-x) / 2} \mathrm{Ni}_{(1-x) / 2} \mathrm{Cr}_{x}$ alloys. Simulation details for computations in the true random alloys are given in Appendix A3. Figs. 11, e show $\Delta E_{X_{0}}^{\text {sol }}$ and $\Delta V_{X_{0}}$ as computed for the $A$-atom and the true random alloys. The accuracy of the $A$-atom approach is consistently very good, particularly with the values being quite small in absolute terms.

Finally, we showcase properties that are extremely difficult to measure in true random alloys: the average interaction energy between a constituent "solute" atom $X_{0}$

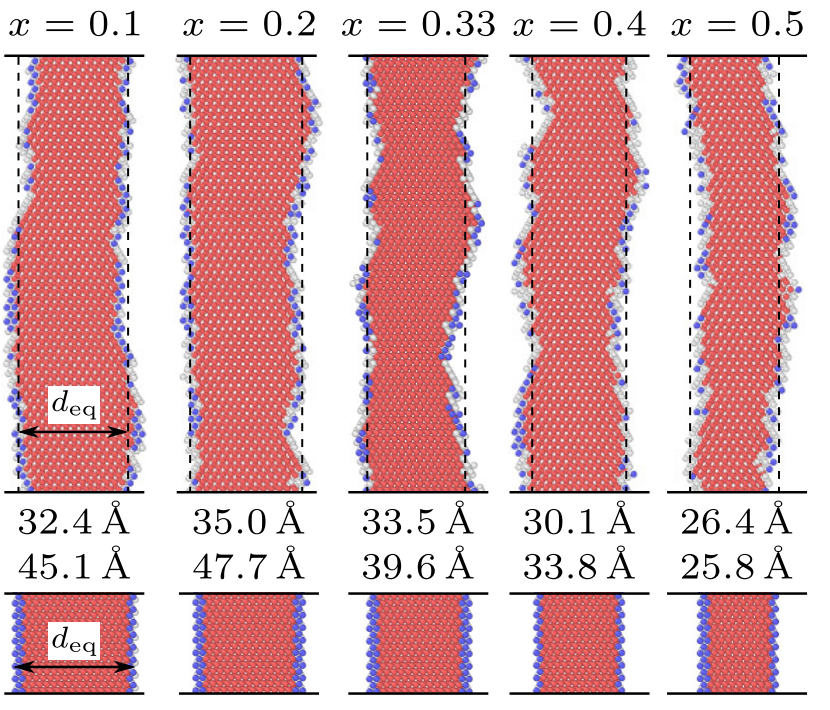

FIG. 3. Dislocation structures and splitting distances $d_{\mathrm{eq}}$ of an edge dislocation in $\mathrm{Fe}_{(1-x) / 2} \mathrm{Ni}_{(1-x) / 2} \mathrm{Cr}_{x}$ alloys vs. $x$, in true random (top) and $A$-atom (bottom) materials. Coloring corresponds to common neighbor analysis 41 : white and violet atoms are associated with the partial cores, and red atoms with the stacking fault. Uncertainty among different realizations in the true random alloys is $\approx \pm 5 \AA$.

and structural defects. We first consider the interaction energy $E_{X_{0}-d}^{\text {int }}$ between an $X_{0}$ atom and an edge dislocation in the multicomponent alloy, as a function of the position of the $X_{0}$ atom. In the true random alloy, the dislocation position itself is not unique because it adjusts to the local fluctuations (see Fig. 3). So, when replacing one other atom type $Y$ by an $X_{0}$, the dislocation adjusts locally; computing $E_{X_{0}-d}^{\text {int }}$ in a true random alloy is thus not clearly defined and is also subject to large fluctuations. Using the $A$-atom potential, however, the average interaction energy at a given position relative to the well-defined, straight, dissociated dislocation in the $A$-atom material is easily computable. Fig. 4 a shows $E_{X_{0}-d}^{\text {int }}$ for $X_{0}=\mathrm{Fe}, \mathrm{Ni}$ and $\mathrm{Cr}$ in the fcc $\mathrm{Fe}_{33} \mathrm{Ni}_{33} \mathrm{Cr}_{33}$ alloy at a wide range of substitutional atomic positions around one of the partial dislocation cores. $\mathrm{Fe}$ and $\mathrm{Ni}$ have repulsive interaction energies just below the partial dislocation, where the dislocation pressure is tensile, consistent with negative misfit volumes (Fig. 11 ), while $\mathrm{Cr}$ shows the opposite behavior. All of these interaction energies are responsible for the high plastic flow strength measured in the true random alloys 40 , and vary with alloy composition. To demonstrate the role of overall composition, we compute the interaction energies of "solutes" with the intrinsic stacking fault $E_{X_{0}-\gamma}^{\text {int }}$ for the $\mathrm{Fe}_{(1-x) / 2} \mathrm{Ni}_{(1-x) / 2} \mathrm{Cr}_{x} A$-atom alloys, and for $X_{0}^{\gamma}$ located in the second plane above the fault plane. Fig. $4 \mathrm{~b}$ shows that the $E_{X_{0}-\gamma}^{\text {int }}$ vary strongly with composition, with the $\mathrm{Cr}$ interaction changing from attractive to repulsive with increasing $\mathrm{Cr}$ content $x$, for instance. In true random alloys, the replacement procedure necessary to compute 


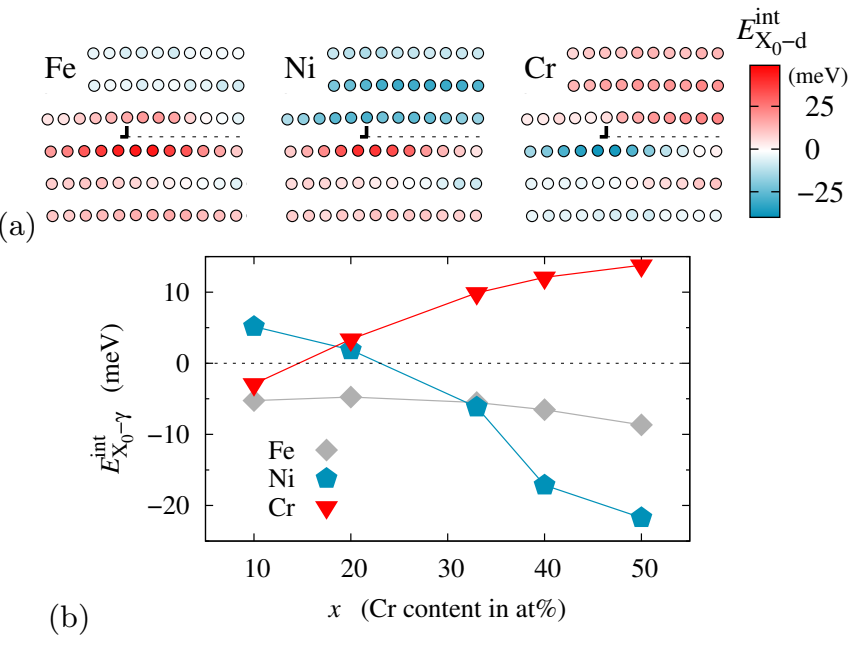

FIG. 4. (a) Interaction energies $E_{X_{0}-d}^{\text {int }}$ between "solutes" $X_{0}=\mathrm{Fe}, \mathrm{Ni}$ and $\mathrm{Cr}$ with an edge dislocation measured in the $\mathrm{Fe}_{33} \mathrm{Ni}_{33} \mathrm{Cr}_{33} A$-atom material. The $\lrcorner$ symbol indicates the left partial and the dashed line the stacking fault trace in the glide plane. (b) Interaction energies $E_{X_{0}-\gamma}^{\text {int }}$ between "solutes" and stacking fault vs. $x$ for all $\mathrm{Fe}_{(1-x) / 2} \mathrm{Ni}_{(1-x) / 2} \mathrm{Cr}_{x} A$-atom alloys.

$E_{X_{0}-\gamma}^{\text {int }}$ involves subtraction of two similar energies with large fluctuations, making determination of the interaction energy inaccurate unless thousands of simulations are run. The $A$-atom approach is thus the only way to measure defect/defect interactions accurately, while also being high efficient and applicable to any multicomponent system.

\section{DISCUSSION}

Having derived the $A$-atom potential and carefully compared its properties to those of true random alloys, we now highlight some of its important features and applications.

Since the configurational averaging in Eqs. 4, 5 and 7 is done on an arbitrary set of atomic sites, using the $A$-atom potential to study structural defects is fully justified, as shown in Figs. 1k, 2p and 3. Energy and forces are analytical for the $A$-atom EAM potential, and thus studies of structural defects present no specific difficulty. This is in contrast to electronic structure CPA approaches to random alloys, where the loss of lattice periodicity in the presence of defects is accompanied by a loss of analyticity that complexifies and/or precludes the solution of the coupled CPA equations $30 / 42 / 43$.

In addition, Eq. 7] shows that it is possible to perform calculations on systems that mix $A$-atoms with real constituent atoms $\{X\}$ to obtain "solute" quantities and interaction energies between any solutes $X_{0}$ in the set $\{X\}$ and any structural defects, in random multicomponent alloys at arbitrary composition, as shown in Figs. 11d,e and 4 . This provides a way to understand how individ- ual constituent elements behave, on average, in the alloy material and how the behavior varies with the overall alloy composition. This also forms a robust basis for developing predictive models of alloy properties 40144 46: the average alloy material is a natural reference state from which one can compute all the necessary model parameters using the $A$-atom potential alone or combined with the elemental potentials.

As we mentioned before, the $A$-atom material has averaged out all the local compositional and structural fluctuations of the true random alloy. By comparing materials properties computed for the $A$-atom and true random alloys, those properties that are controlled by the average and by the fluctuations, respectively, are clearly identified. This is physically insightful and valuable for understanding the mechanistic origin of a given material property.

Next, let us state again that the $A$-atom potential is a new EAM potential. Starting from a known set of potentials for elements $\{X\}$, the $A$-atom potential for a targeted random alloy is fully defined by Eqs. 5. This will have great benefits in developing/benchmarking potentials for multicomponent alloys, especially for modeling mechanical properties. Indeed, the $A$-atom potential allows for fast and accurate sampling of many bulk and defect properties for the whole compositional space, which can be very large for some multicomponent systems such as High Entropy Alloys 4748 . Moreover, from a known set of potentials, an infinite number of new potentials can be generated and explored using the $A$-atom approach. For instance, a family of potentials with controlled materials properties can be created, in which only one property varies while the other ones are held nearly fixed. This can permit careful study of the role of individual material properties on macroscopic behavior, e.g. the role of stacking fault energy on the strength of nanocrystalline metals 5 . More subtle is the fact that it can lead to better potentials for desired elemental materials. Several preexisting EAM potentials for the same pure element can be combined with different weights (as represented by the "concentrations") to generate a new potential with an improved description of the elemental properties.

Finally, the $A$-atom potential should be useful in concurrent multiscale modeling methods, extending atom/continuum multiscale models such as the Quasicontinuum 49 51 or the Coupled Atomistic DiscreteDislocation $\frac{52[53}{m}$ models to random alloys. Specifically, the $A$-atom potential can be used efficiently and accurately to compute the material properties in coarsegrained continuum regions using the Cauchy-Born rule, with the compositional averaging performed automatically and with the ability to refine the description down to the atomic scale. 


\section{CONCLUSION}

In summary, we have presented and validated the $A$ atom potential as an efficient and elegant way to compute many average properties of random alloys, including defect properties. In particular, we have demonstrated the valuable use of the $A$-atom method in computing defect/defect interactions that are essentially unobtainable by direct simulations on random alloys. We have further identified a host of very useful application concepts for the $A$-atom approach. In future work, we will report on specific applications to problems in plastic flow stress, cross-slip, and fracture of complex metal alloys, and further show that the $A$-atom approach accurately captures the finite-temperature thermodynamic properties of true random alloys 54 .

\section{ACKNOWLEDGMENTS}

Support for this work was provided through a European Research Council Advanced Grant, "Predictive Computational Metallurgy", ERC Grant agreement No. 339081 - PreCoMet.

\section{Appendix A: Simulation details for defect properties}

In this section, important numerical details about the calculation of defect properties into the true random alloys are provided.

\section{Planar defects}

Intrinsic stacking fault energies are computed using simulation boxes of size $x \times y \times z=50 \times 50 \times 23$ along the $[10 \overline{1}],[1 \overline{2} 1]$ and [111] directions. Periodic boundary conditions are applied along $x$ and $y$ directions, and free surface conditions along the $z$ direction. This simulation size is large enough to both prevent any interaction between the fault and the free surfaces, and to obtain a large enough sampling of the random composition in the fault plane. Similar simulation size ( $>300000$ atoms) and boundary conditions are used for the surface energy calculations of the (001) and (111) planes.

\section{Dislocations}

The average dislocation configuration is extracted from true random alloys as follows. Random distributions of atoms at the targeted compositions are prepared on simu-

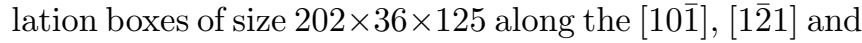
[111] directions (5454000 atoms). Then, a $\{111\}(110)$ edge dislocation is introduced by adding an extra atomic plane, that is spread over the expected equilibrium dissociation distance, as given into the $A$-atom material.
Periodic boundary conditions are applied along $x$ and $y$ directions, and free surface conditions along the $z$ direction. Relaxation at $0 K$ is performed, and then the average dissociation distance is measured by averaging over 20 different random realizations for each alloy composition.

\section{Substitutional constituent solutes}

Calculations of solution energies and misfit volumes are performed on simulations of $6 \times 6 \times 6$ fcc unit cells (864 atoms). We exemplify the exact computational procedure in the true random alloys on the misfit volume calculation. The average misfit volume of the $X_{0}$ species, $\Delta V_{X_{0}}$, is computed using a replacement procedure, where each atom of the random atomic configuration is replaced by an $X_{0}$-type atom, and the associated relaxation volume $\Delta V_{X_{0}}^{n}$ is measured $\left(n \in\left[1, N_{s}\right]\right.$ labels the atomic sites, here). A collection of measurements is then obtained for each $X_{0}$. This raw dataset can be subdivided into different datasets $\left\{\Delta V_{X_{0}}^{k_{Y}}\right\}$, with $k_{Y} \in\left[1, n_{Y}\right]$, where $\Delta V_{X_{0}}^{k_{Y}}$ corresponds to the relaxation volume resulting from the replacement of a $Y$-type atom located in the $k_{Y}$ site by an $X_{0}$-type atom, and $n_{Y}=c_{Y} N_{s}$ with $c_{Y}$ the concentration in $Y$ atoms. The average misfit volume of $X_{0}$ can thus be written as

$$
\begin{aligned}
\Delta V_{X_{0}} & =\frac{1}{N_{s}} \sum_{n=1}^{N_{s}} \Delta V_{X_{0}}^{n}, \\
& =\frac{1}{N_{s}} \sum_{Y=1}^{N} \sum_{k_{Y}=1}^{n_{Y}} \Delta V_{X_{0}}^{k_{Y}}, \\
& =\sum_{Y=1}^{N} c_{Y} \Delta V^{X_{0} / Y},
\end{aligned}
$$

with $\Delta V^{X_{0} / Y}=\frac{1}{n_{Y}} \sum_{k_{Y}=1}^{n_{Y}} \Delta V_{X_{0}}^{k_{Y}}$, and $\Delta V^{X_{0} / X_{0}}=$ 0 . The resulting misfit volumes satisfy the sum rule $\sum_{X_{0}=1}^{N} c_{X_{0}} \Delta V_{X_{0}}=0$. The computation of the average misfit volume of one constituent element in the $\mathrm{Fe}_{33} \mathrm{Ni}_{33} \mathrm{Cr}_{33}$ alloy, with the 864 atoms simulation size, thus requires 576 calculations, whereas the $A$-atom method requires only a single computation. The computational benefit of the $A$-atom approach is obvious, especially when the number of alloying components increases.

Note that the raw dataset obtained from true random alloy calculations shows fluctuations around the average value of each misfit volume, that can be rather important for some alloy compositions. Large fluctuations of some point defect properties with the local chemical environnment - without attempt to reach the average property have also been observed by some authors by DFT calculations in concentrated alloy $\$ 55156$.

Attempts to obtain interaction energies between "solutes" $X_{0}=\mathrm{Fe}, \mathrm{Ni}$ and $\mathrm{Cr}$ and a stacking fault $E_{X_{0}-\gamma}^{\text {int }}$ 
were made for the equicomposition fcc $\mathrm{Fe}_{33} \mathrm{Ni}_{33} \mathrm{Cr}_{33}$ alloy. A random distribution of atoms at the equicomposition is prepared on a simulation box of size $50 \times 50 \times 23$ along the $[10 \overline{1}],[1 \overline{2} 1]$ and $[111]$ directions, that contains a stacking fault. For each "solute" $X_{0}, 30$ different $Y$ in the atomic plane one plane above the fault plane are successively replaced by an $X_{0}$. This is repeated for all $\{Y\} \neq X_{0}$ and the associated average energy is computed. This does not permit a determination of $E_{X_{0}-\gamma}^{\text {int }}$ with good precision: there are large fluctuations in energy among the different individual measurements, and the final interaction values are very small (lower than $8 \mathrm{meV}$ for the equicomposition alloy). Increasing the sampling to the same number of replacements as for the misfit volume calculation did not significantly improve the accuracy of the measurement. Thus, instead of the one calculation needed per solute/stacking interaction in the $A$-atom material, thousands of calculations would be necessary to obtain only one solute/stacking interaction in the true random alloy with an acceptable accuracy. This is not a tractable approach to capture defect/defect interactions over a range of alloy composition in multicomponent alloys.
* Celine.Varvenne@epfl.ch Corresponding author

1 M. Kabir, T. T. Lau, D. Rodney, S. Yip, and K. J. Van Vliet, Phys. Rev. Lett. 105, 095501 (2010).

2 M.-C. Marinica, F. Willaime, and J.-P. Crocombette, Phys. Rev. Lett. 108, 025501 (2012)

3 W. A. Curtin, D. L. Olmsted, and L. G. H. Jr, Nat. Mater. 5, $875(2006)$

${ }^{4}$ M. Diarra, A. Zappelli, H. Amara, F. Ducastelle, and C. Bichara, Phys. Rev. Lett. 109, 185501 (2012)

5 V. Yamakov, D. Wolf, S. R. Phillpot, A. K. Mukherjee, and H. Gleiter, Nat. Mater. 3, 43 (2004)

${ }^{6}$ M. S. Daw and M. I. Baskes, Phys. Rev. B 29, 6443 (1984).

7 M. W. Finnis and J. E. Sinclair, Philos. Mag. A 50, 45 (1984).

8 V. Rosato, M. Guillopé, and B. Legrand, Philos. Mag. A 59, $321(1989)$.

9 M. I. Baskes, Phys. Rev. B 46, 2727 (1992)

10 A. Caro, D. A. Crowson, and M. Caro, Phys. Rev. Lett. 95, $075702(2005)$

${ }^{i 1}$ F. Apostol and Y. Mishin, Phys. Rev. B 83, 054116 (2011)

12 G. P. P. Pun and Y. Mishin, Phys. Rev. B 86, 134116 (2012)

${ }^{13}$ M. Cak, T. Hammerschmidt, J. Rogal, V. Vitek, and R. Drautz, J. Phys.: Condens. Matter 26, 195501 (2014).

14 J. Gehrmann, D. G. Pettifor, A. N. Kolmogorov, M. Reese, M. Mrovec, C. Elsässer, and R. Drautz, Phys. Rev. B 91, 054109 (2015)

1o W. J. Szlachta, A. P. Bartók, and G. Csányi, Phys. Rev. B 90, 104108 (2014)

io S. Huygh, A. Bogaerts, A. C. van Duin, and E. C. Neyts, Comput. Mater. Sci. 95, 579 (2014).

17 B.-J. Lee and M. I. Baskes, Phys. Rev. B 62, 8564 (2000)

18 B.-J. Lee, M. I. Baskes, H. Kim, and Y. K. Cho, Phys. Rev. B 64, 184102 (2001)

${ }^{19}$ Z. Wu, M. F. Francis, and W. A. Curtin, Modelling Simul. Mater. Sci. Eng. 23, 015004 (2015).

${ }^{20}$ R. W. Smith and G. S. Was, Phys. Rev. B 40, 10322 (1989)

21 M. Grujicic and X. Zhou, Calphad 17, 383 (1993)

${ }^{22}$ R. Najafabadi, H. Y. Wang, D. J. Srolovitz, and R. Lesar, Acta Metall. Mater. 39, 3071 (1991)

23 R. Smith, R. Najafabadi, and D. Srolovitz, Acta Metall. Mater. 43, 3621 (1995)

${ }^{24}$ M. Grujicic and X. Zhou, Mater. Sci. Eng.: A 190, 87 (1995)

25 M. Grujicic, J. Mater. Sci. 32, 1749 (1997)

26 L. Nordheim, Ann. Phys. 607 (1931).
${ }^{27}$ R. J. Elliott, J. A. Krumhansl, and P. L. Leath, Rev. Mod. Phys. 46, 465 (1974)

28 J. Faulkner, Prog. Mater. Sci.s 27, 1 (1982).

29 D. D. Johnson, D. M. Nicholson, F. J. Pinski, B. L. Gyorffy, and G. M. Stocks, Phys. Rev. Lett. 56, 2088 (1986)

${ }^{30}$ F. Ducastelle, Order and Phase Stability in Alloys (NorthHolland, Amsterdam, 1991).

31 D. Johnson and M. Asta, Comput. Mater. Sci. 8, 54 (1997), proceedings of the joint NSF/CNRS Workshop on Alloy Theory.

32 B. Ujfalussy, J. S. Faulkner, N. Y. Moghadam, G. M. Stocks, and Y. Wang, Phys. Rev. B 61, 12005 (2000)

33 A. Taga, L. Vitos, B. Johansson, and G. Grimvall, Phys. Rev. B 71, 014201 (2005).

34 The second order term contribution on the total energy was computed $\sqrt{22}$ for some bulk materials, and was found to be negligible.

35 Eq. 4 is thus gauge invariant, e.g. applying the gauge transformation $\tilde{F}^{A}=F^{A}+k \bar{\rho}_{i}$ and $\tilde{V}_{i j}^{A A}=V_{i j}^{A A}-2 k \rho_{i j}^{A}$, with $k$ a constant and $\rho_{i j}^{A}=\sum_{X} c_{X} \rho_{i j}^{X}$.

36 G. Bonny, D. Terentyev, R. C. Pasianot, S. Ponce, and A. Bakaev, Modelling Simul. Mater. Sci. Eng. 19 (2011), 10.1088/0965-0393/19/8/085008.

37 S. Plimpton, J. Comput. Phys. 117, 1 (1995).

38 J. P. Hirth and J. Lothe, Theory of Dislocations, 2nd ed. (Wiley, New York, 1982).

39 J. R. Rice, J. Mech. Phys. Solids 40, 239 (1992)

40 C. Varvenne, A. Luque, and W. A. Curtin, submitted (2015).

41 A. Stukowski, Modelling Simul. Mater. Sci. Eng. 18, $015012(2010)$

${ }^{42}$ P. Turchi, G. Treglia, and F. Ducastelle, J. Phys. F: Metal Physics 13, 2543 (1983)

43 G. Treglia, B. Legrand, and F. Ducastelle, EPL (Europhysics Letters) 7, 575 (1988).

44 G. P. M. Leyson, W. A. Curtin, L. G. Hector, Jr., and C. F. Woodward, Nat. Mater. 9, 750 (2010).

45 J. A. Yasi, L. G. Hector Jr, and D. R. Trinkle, Acta Mater. 58, 5704 (2010).

${ }^{40}$ A. Luque, M. Ghazisaeidi, and W. A. Curtin, Acta Mater. 81, 442 (2014)

${ }^{47}$ M.-H. Tsai and J.-W. Yeh, Mater. Res. Lett. 2, 107 (2014)

48 D. B. Miracle, J. D. Miller, O. N. Senkov, C. Woodward, M. D. Uchic, and J. Tiley, Entropy 16, 494 (2014)

49 V. B. Shenoy, R. Kukta, and R. Phillips, Phys. Rev. Lett. 84, $1491(2000)$ 
50 L. M. Dupuy, E. B. Tadmor, R. E. Miller, and R. Phillips, Phys. Rev. Lett. 95, $060202(2005)$.

51 V. Sorkin, R. S. Elliott, and E. B. Tadmor, Modelling Simul. Mater. Sci. Eng. 22, 055001 (2014)

${ }^{52}$ L. E. Shilkrot, R. E. Miller, and W. A. Curtin, Phys. Rev. Lett. 89, $025501(2002)$
53 L. Shilkrot, R. E. Miller, and W. A. Curtin, J. Mech. Phys. Solids 52, 755 (2004)

${ }^{54}$ W. G. Nöhring and W. A. Curtin, submitted (2015).

55 T. P. C. Klaver, R. Drautz, and M. W. Finnis, Phys. Rev. B 74, 094435 (2006).

5o J. B. Piochaud, T. P. C. Klaver, G. Adjanor, P. Olsson, C. Domain, and C. S. Becquart, Phys. Rev. B 89, 024101 $(2014)$ 